\title{
16 Social Media Collaboration in Software Projects
}

\author{
Rachel Harrison and Varsha Veerappa
}

\begin{abstract}
Social media has had a big impact on the way that software projects are managed and the way that stakeholders interact with each other: indeed, the nature of software projects has evolved substantially in keeping with the evolution of technology. A direct consequence of the ubiquity of the internet is the increasing trend towards cooperation outside the boundaries of an office. The interactions involved in software projects have changed accordingly and can be broadly divided into two types (1) interactions among stakeholders who are in a single location, (for example people sharing the same office space) and (2) interactions among stakeholders who are in distributed locations (for example software projects which are partly implemented offshore). Social media has been and remains a significant facilitator to these kinds of interactions. This chapter looks at the implications of the use of social media in 21 st Century software projects.
\end{abstract}

\subsection{Introduction}

We use the term social media to include all web-based platforms that allow the creation and exchange of user generated content using Web 2.0 [1]. Social media can be used in many circumstances. These include collaborative projects like wikis where a group of individuals come together to share knowledge with the idea that the effort of all the users together gives a better outcome than that of a single user. An example of a very popular collaborative project is Wikipedia1. Another example of social media is the blog in which a single user contributes to content but allows other users to interact with features such as comments and likes. Users can create these blogs easily on specialized platforms like Tumblr2, Blogger3 or Wordpress4. Microblogs are very similar to blogs and enable users to post very short entries as brief updates. An example of a very popular microblog platform is Twitters.

\footnotetext{
1 www.wikipedia.org

2 www.tumblr.com

3 www.blogger.com/

$4 \mathrm{http}: / /$ wordpress.com/

5 https://Twitter.com/
} 
Social networking sites such as Facebook6 and Google+7 enable users to create personal profile pages (with information such as photos, videos and blogs) and connect to other users to share their profile information. The users can also further interact with each other via email and instant messengers that are integrated into those social networking sites. Such sites also allow organisations to create their own pages which can be used to promote awareness about their services and activities. Professional networking sites such as LinkedIns are very similar. They enable users to post information about their careers and network with other users with similar interests. Other forms of social media include virtual games and social worlds such as SecondLife9 and content communities such as YouTube10.

The popularity of Social Media continues to increase. In October 2012, Facebook estimated its number of users to be 1 billion while the number of Tumblr blogs is 79.3 million. Twitter on the other hand had more than 500 million users registered as at March 2013. People are now using social media both informally for personal social interactions and formally for business and work. Indeed social media provide an efficient and instantaneous means of communication that can be very useful to the health services [1-3], marketing[4], education [5] and governments [6]. One sector that has particularly benefited from the use of social media is the software development industry.

In recent years, software development has evolved from a mainly closed in-house activity to a more open and globally distributed one. This new context has given rise to new challenges such as communication, synchronous and asynchronous coordination and trust [7]. One way to address these problems is to use social media to facilitate communication, task synchronisation and knowledge sharing. This can be done at two levels: the project level (where software teams, domain experts and clients interact with each other to ensure a successful outcome) and the community level where the software team interacts with peers in the broader community of software professionals to share knowledge.

In this chapter we look at how the three main groups of people who are directly or indirectly involved in a project (internal stakeholders, external stakeholders and peers) interact. We have carried out a pilot study with practitioners in industry to understand how social media is used in their organisations for the different types of interactions we identified. The results of the study are used as evidence to support the points we make in this chapter.

\footnotetext{
$6 \mathrm{http}: / /$ www.facebook.com/

$7 \mathrm{https}: / /$ plus.google.com/

8 www.linkedin.com

$9 \mathrm{http}: / /$ secondlife.com/

$10 \mathrm{http}: / /$ www.youtube.com
} 


\subsection{Interactions in Software Projects}

The three main groups of people involved in a project are internal stakeholders, external stakeholders and peers. External stakeholders are the individuals or organisations that are not part of the organisation doing the actual software development activities but who have an influence on the decisions in the projects. They are not usually in the same location as the internal stakeholders. For example, an external stakeholder could be a manager, an end-user in the client organisation or a domain expert. Such stakeholders are usually involved in the requirements elicitation and elaboration processes as well as during testing. Figure 1 depicts the various types of interactions among these stakeholders during a typical software project.

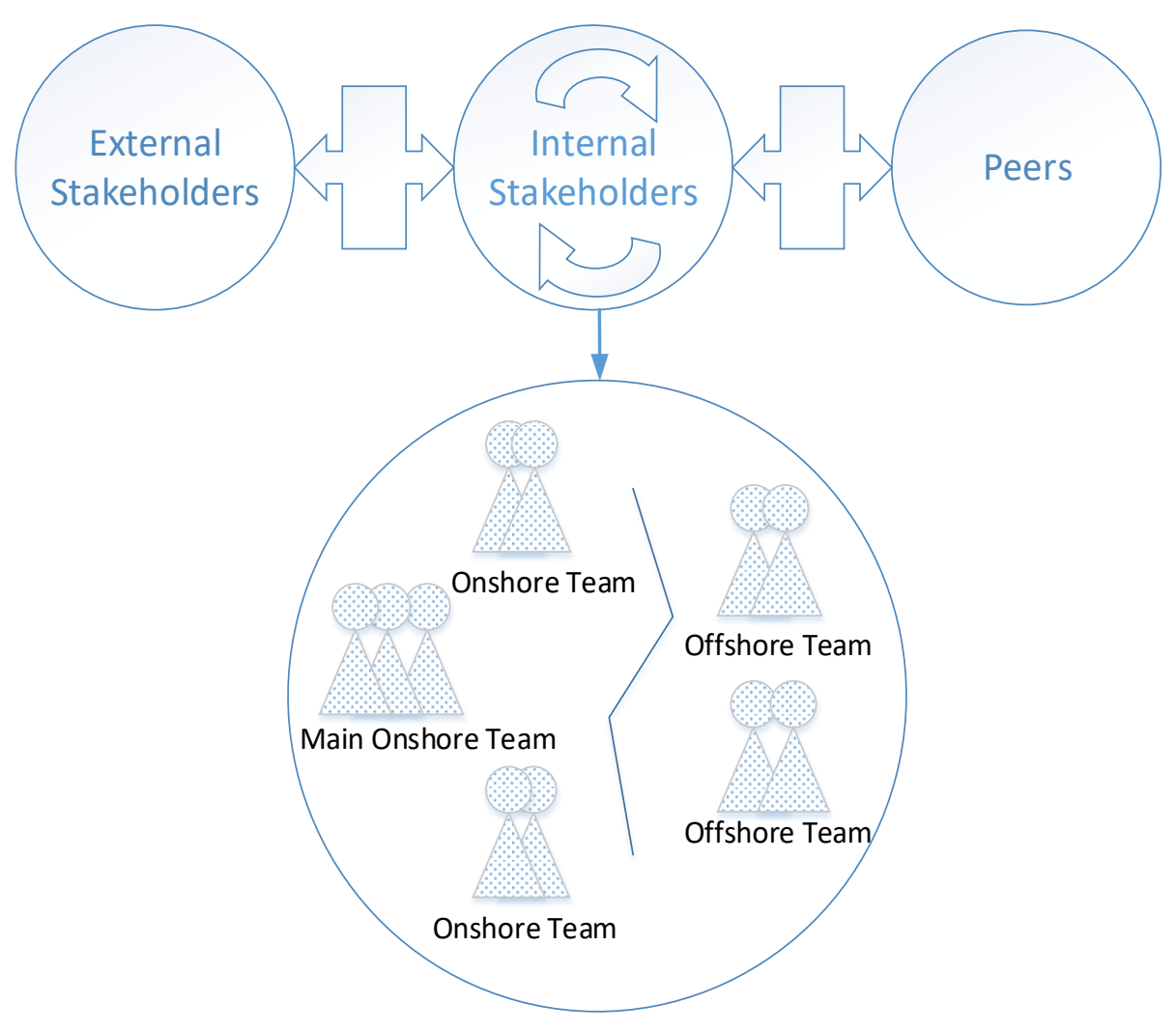

Fig. 1. Types of Interactions in Software Projects

Internal stakeholders are individuals or teams within the same company that interact on a daily basis during the software development process. These stakeholders can be co-located or they could be distributed geographically in the same country or across the 
world. In a software project, there is usually a project manager in charge of the main team where the project decisions are taken. There may also be other teams that are located in the same country as the main team. Some of the tasks may also be outsourced to offshore teams. All these stakeholders are internal stakeholders. Software engineers, project managers and testers are examples of such stakeholders. Their involvement in software projects range from requirements elicitation through to design, implementation and testing.

Peers are individuals who are known to the internal stakeholders either through previous acquaintance or through necessity for the project. They usually share their knowledge and experiences and even refer new peers to the internal stakeholders. Peers are usually only indirectly involved in a software project. They can provide feedback and solutions to technical problems that the internal stakeholders encounter in the software projects they are working on.

\subsection{Social Aspects of Software Projects}

The very nature of software projects necessitates social behaviours. According to Begel et al. [8], the model of teaming from Tuckman and Jensen [9] can be applied to the software lifecycle to explain the social aspects of software projects. During the early phases of a software project, internal stakeholders organise into teams. This is known as forming. They then need to come to consensus about their goals through storming. They then choose and implement their software methodology and engineering processes which is known as norming. Stakeholders collaborate and coordinate the tasks among themselves to create a new product through performing. At the end of the project, adjourning enables them to reflect on their successes and failures in order to improve the next project's execution and outcome.

Interactions between internal and external stakeholders can involve the same social interaction and processes. These interactions may not always be directly related to coding and implementation. The external stakeholders are identified by the internal stakeholders through forming and storming during which the requirements of the external stakeholders are elicited and prioritised or milestones are agreed. During performing external stakeholders test the deliverables to determine if they have met their expectations and the adjourning process involves any post-implementation reporting that helps to determine the success or failure of a project.

Internal stakeholders and peers interact mostly for the exchange of ideas and current practices and these interactions usually occur continuously and are not restricted by a specific software project. However, these interactions are more likely to occur during the design and implementation phases of the software development life cycle. During these interactions, peers and internal stakeholders exchange knowledge on best practice, technical issues and latest trends in their fields of interest. This can be a great motivating factor for software projects (see Chapter 12 (Beecham)). 
These interactions that enable and facilitate the software development activities require the stakeholders to be able to find and connect with other stakeholders who have similar aims in the project or who have complementary skills.

\subsection{Importance of Social Media in Software Projects}

The nature of software projects has changed over time. Development is now often done in teams which are both onshore and offshore. In 2008, the total global IT outsourcing was estimated to be worth between $\$ 220$ billion and $\$ 250$ billion with a forecasted growth of 6-9\% per annum to reach around $\$ 380$ billion by 2013 [10]. This emphasises the need for successful completion of projects as the incurred losses in case of failure can be considerable. Social media are one of the key enablers for any projects that involve distributed teams in general.

\subsection{Pilot Study}

The pilot study was conducted over a period of two months with nine companies. We contacted managers who had an overview of the software development process in their respective companies. Data collection was performed using a structured questionnaire with both open-ended and close-ended questions. Each individual had to answer sixteen questions about their past and current use of social media in software projects as well as the software development methodologies that are used in their companies. The questionnaire is included in Figure 2.

\subsubsection{Data Collection and Analysis}

Our underlying motivation was to investigate the use of social media in global software projects. We wanted to find out what kind of social media is being used and how it is being used to better understand the evolution of social media and to predict future trends. The participants were practitioners drawn from 9 different organizations as shown in Table 1. The participants were all involved in global software development and included managing directors, project managers and software engineers.

The organizations were chosen because they were known to perform software engineering projects and to use social media. Initially the organizations were contacted by email to establish their suitability and willingness to participate. The participants were sent the questionnaire by email and were given 2 months in which to complete it. The participants were all experienced in collaborating on projects and using social media. 
The aim of this questionnaire is to determine how Social Media are currently being used in Software Projects. We want to understand the trends in the use of Social Media and which activities in software projects Social Media facilitate. Examples of social media include Twitter, Facebook, Forums, Chat Clients and Blogs. These can be either public (such as a blogging site that is accessible by everybody on the internet) or private (such as a forum being hosted on company intranet for example).

1 Evolution of the use of Social Media with time

1.1 For how long have you been using Social Media in software projects?

1.2 Which Social Media you have used?

1.3 Which Social Media do you use now?

1.4 If you have stopped using some Social Media, why have you done so in each case?

1.5 Do you think that social media have increased productivity in projects? Why?

2 Activities facilitated by Social Media

2.1 Which activities of the Software Projects are facilitated with Social Media?

1. $\quad$ Requirements Elicitation $\square$

2. $\quad$ Stakeholder Identification/Recommendation $\square$

3. Design $\square$

4. Implementation $\square$

5. $\quad$ Testing $\square$

6. $\quad$ Maintenance $\square$

2.2 Which type of Social Media do you use in each case?

2.3 How essential are Social Media for each the activities you have mentioned?

3 Context of use of Social Media

3.1 Do you use Social Media to interact with colleagues in the same location as you?

3.1.1 If yes, which activities do you use Social Media for?

3.2 Do you use Social Media to interact with colleagues in a different location from you?

3.2.1 If yes, which activities do you use Social for?

3.3 Do you use Social Media to interact with peers who are not your current colleagues?

3.3.1 If yes, which activities do you use Social Media for?

$4 \quad$ Software Methodologies and Social Media

4.1 Does your company use prescribed software methodologies?

4.1.1 If yes, how essential is the use of social media in those software methodologies?

Fig. 2. Pilot study questionnaire 
Table 1. Characteristics of participating organisations

\begin{tabular}{|c|l|c|}
\hline $\begin{array}{l}\text { Organiza- } \\
\text { tion }\end{array}$ & Application Domain & $\begin{array}{l}\text { No. of staff on site (Ap- } \\
\text { prox.) }\end{array}$ \\
\hline A & IT standardization and certification & 5 \\
\hline B & Automobile R\&D & 85 \\
\hline C & Software services & 20 \\
\hline D & Financial services & 40 \\
\hline E & Software services & 9 \\
\hline F & $\begin{array}{l}\text { Socio-technical systems develop- } \\
\text { ment }\end{array}$ & 200 \\
\hline G & Management consulting services & over 500 \\
\hline H & Software services & 15 \\
\hline I & Software services & 40 \\
\hline
\end{tabular}

Data thus received from the participants was anonymised and encoded to extract information that was relevant to the study. We performed a qualitative analysis of the ensuing data to look for patterns of behaviour and unexpected behaviour as well as confirmations of expected behaviour as far as the use of social media in software projects is concerned.

\subsubsection{Results: Social Media and Software Projects}

Social media provide many tools to support the processes involved in executing a software project. Social media can aid software development activities from the early phases such as requirements engineering through to development, testing and documentation. They also provide a means to facilitate maintenance activities. Since social media are highly flexible, stakeholders can readily adapt them to fit their needs to help deliver successful software projects. Our survey shows that all the phases of the software development life cycle can involve the use of social media as illustrated in Figure 3.

Two main characteristics of social media determine how they are used in software projects. These are social presence [11] and media richness [12]. Social presence is the acoustic, visual, and physical contact that users can achieve using social media; that is, it is the level of awareness that users have of each other during communication. Social media with high social presence support synchronous and personal interactions and increase the influence that the users have on each other. Social platforms that enable live web chats are examples of such social media. On the other hand, social media with low social presence favour asynchronous and more formal interactions [13]. Forums are a good example of such social media. 


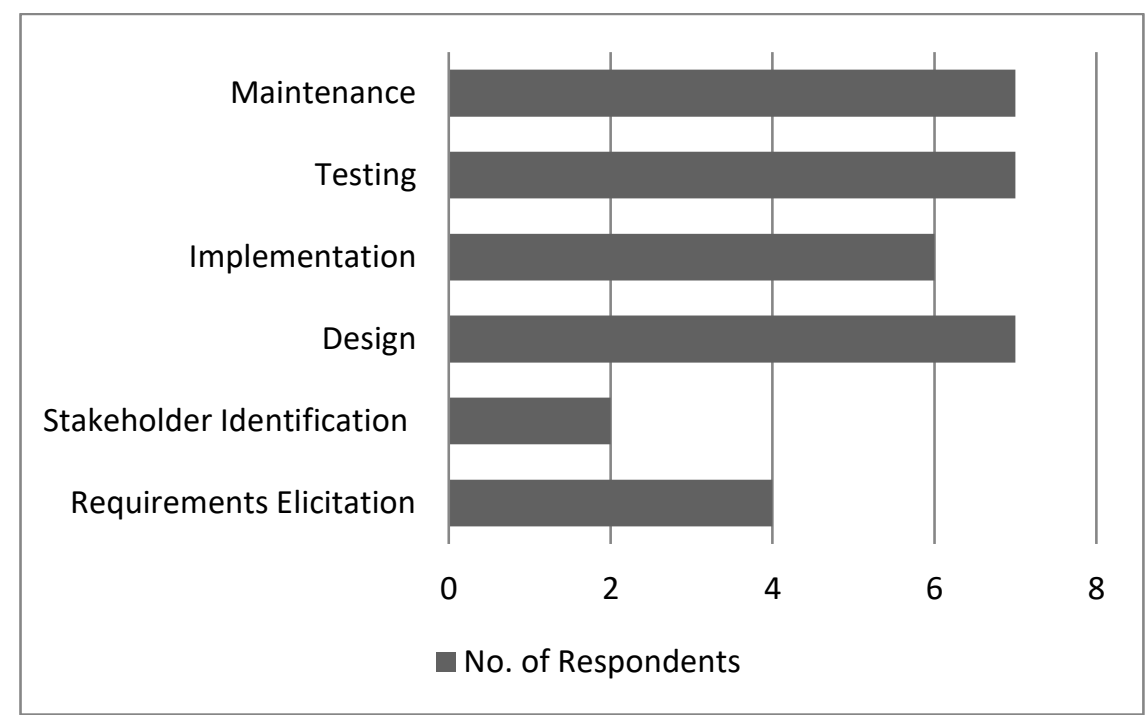

Fig. 3. Use of Social Media in the Software Project Lifecycle

Media richness is the volume of information social media can exchange in a given time interval. Thus, social media with high media richness will use a number of cues to increase clarity of the information being exchanged and actively facilitate feedback. High media richness therefore implies low ambiguity and uncertainty in the information being transmitted. For example, explaining a solution via a web chat is likely to have lower ambiguity and uncertainty than explaining it using posts on a forum or by email. Social presence and media richness determine the appropriateness of a given social media in the different stages of the software development life cycle.

From our survey, we found that although social media have been used in software projects since the introduction of web 2.0, there has been a relatively high rate of adoption of social media in the last decade, similar to that which occurred at the time of the birth of popular social media platforms such as Wikipedia, Facebook and Twitter. The most popular social media used include (unsurprisingly) Facebook, Twitter, instant messengers, LinkedIn and blogs. Figure 4 shows our findings on the use of different social media in the software project lifecycle.

We found that currently the design, testing and maintenance phases top the list for the use of social media with the largest variety of social media being involved during design. Social media use is least popular in stakeholder identification/recommendation and requirements elicitation activities. Software teams use Skype ${ }^{\mathrm{TM}}$ and forums in all phases of the software projects they are involved in. Twitter tends to be used in maintenance, stakeholder identification and recommendation, design and maintenance while instant messengers and blogs tends to be used during design, implementation, testing and maintenance. Facebook is used only to elicit requirements and stakeholder analysis while LinkedIn has been identified as being useful mainly in the implementation phase 
of software projects. An interesting observation here is that although requirements elicitation and stakeholder identification/recommendation activities involve a lot of interaction among stakeholders, they lag behind in the adoption of social media.

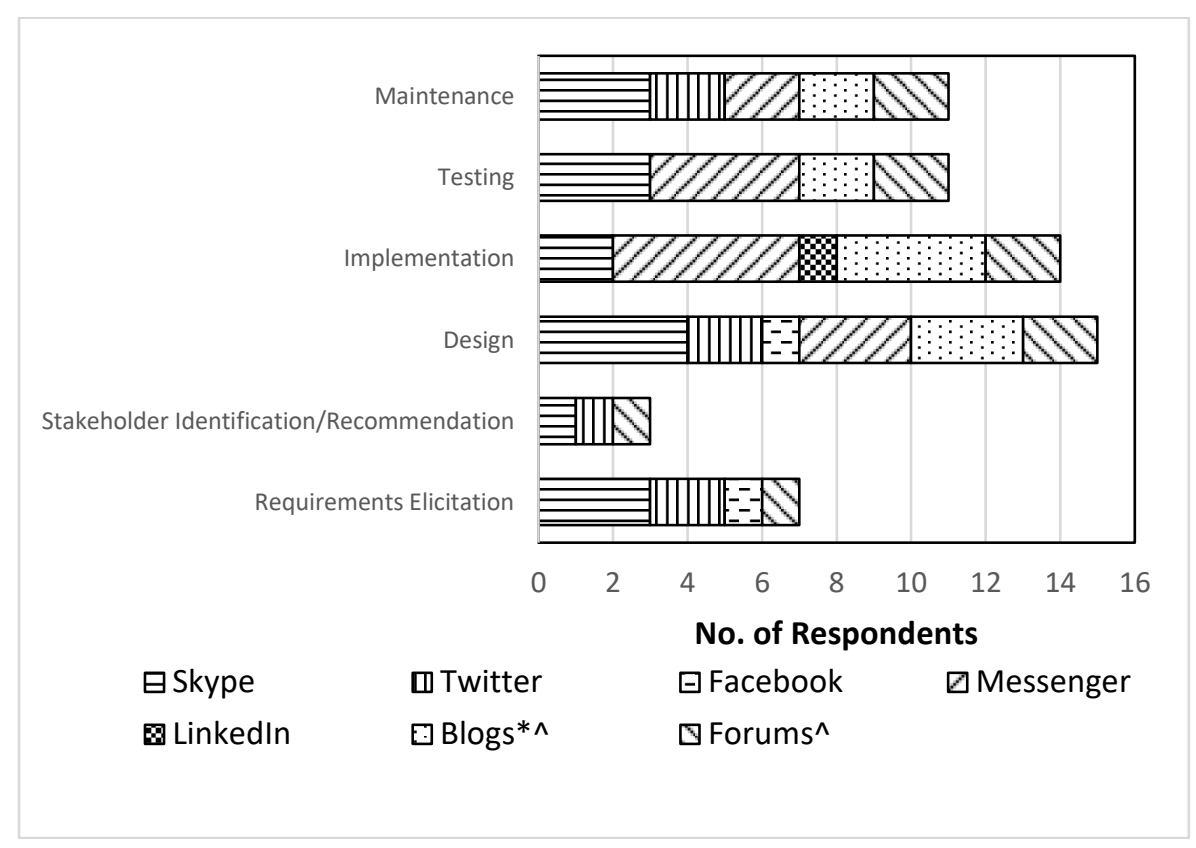

Fig. 4. Types of Social Media used in the different phases of Software Projects

The popularity of instant messaging in general and $\mathrm{Skype}^{\mathrm{TM}_{11}}$ in particular which provide both high social presence and high media richness can be explained by the fact that some of the tasks during software projects require very intense interactions among the stakeholders. One such example is when developers need a quick solution to a critical technical problem they encounter during implementation; they may need and want to discuss the problem urgently in detail and interact with technical experts in the team.

Forums enable stakeholders and peers to communicate asynchronously with a low level of formality and interaction. Thus stakeholders tend to use these in situations which are not urgent. For example, forums are very useful for explaining generic solutions to recurring problems which can be used by other software teams within an organization.

Twitter is actively used to exchange information between stakeholders in software projects because it provides support for lightweight coordination and communication. Although the social presence and media richness is limited, the broadcast mechanisms implemented in Twitter are very efficient in reaching a large audience.

11 http://www.skypeTM.com/en/ 
Blogs are frequently used by stakeholders and peers to document technical information, to discuss the release of new features and to support requirements engineering. Blogs are useful in these cases because they encourage discussions and are accessible by a large number of people who can participate whenever they have time to do so.

Facebook and LinkedIn allow the creation of virtual communities in which stakeholders and peers can share views and information about specific topics related to software projects. Facebook can help stakeholders to recommend other stakeholders by inviting them to the software project pages.

\subsubsection{Results: Interaction among co-located stakeholders}

Stakeholders who are co-located may use social media to communicate with each other despite being in the same location. This is particularly likely for large organisations with many large teams. Here, stakeholders use social media to keep up-to-date with what other teams are doing and to facilitate communication during software projects. Figure 5 illustrates how participants in our survey use social media to interact with co-located stakeholders.

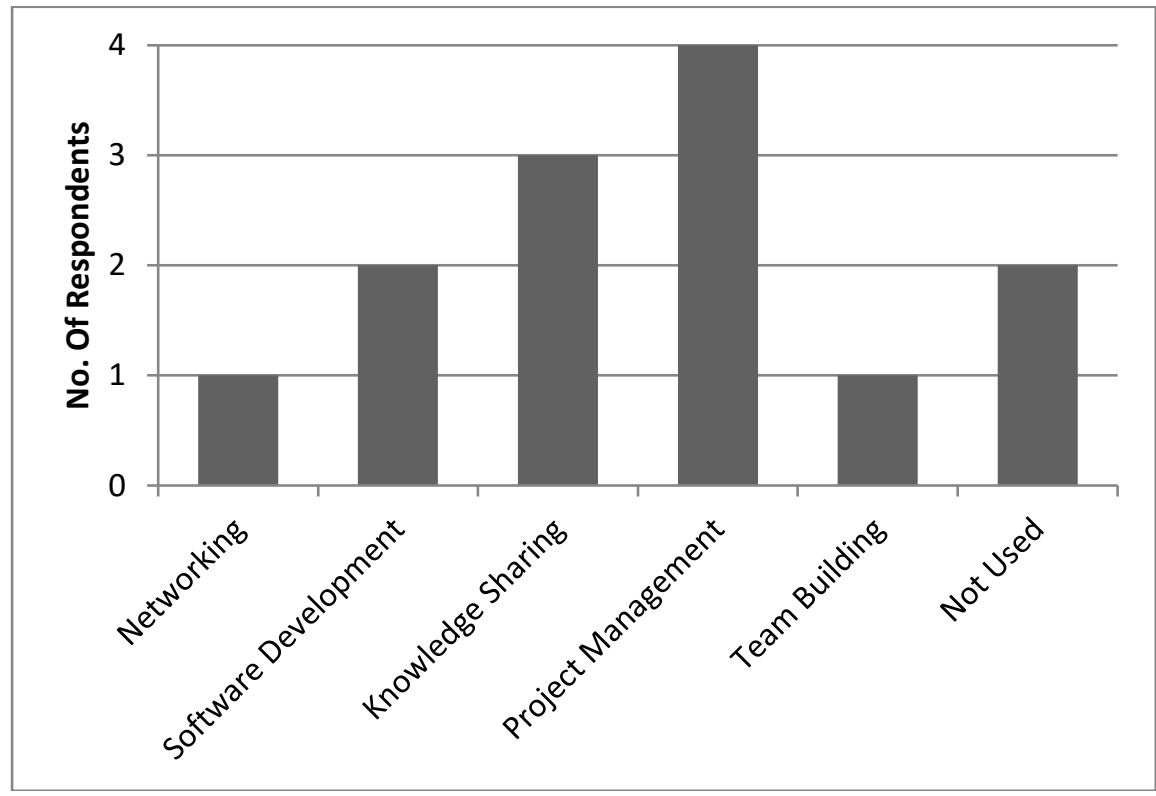

Fig. 5. Types of Interactions among Co-located Stakeholders

Figure 5 shows that social media is not always used among co-located teams. However, when it is used, it predominantly supports project management activities. Senior team members tend to use social media platforms like wikis or instant messaging for 
tracking the progress of tasks in a project. Wikis are excellent facilitators of software development collaboration. Their adoption in software projects is widespread. Wikis are mainly used to support defect tracking, documentation, requirements tracking, and test case management and to create project portals. Tagging is used in software project portals such as Github 12 to tag issues, project releases, work items and builds. The main advantages of using tags in software projects are their flexibility and their lightweight, bottom-up nature.

Instant messengers are extensively used among team members for coordination of tasks. For example, in our study, we found that in projects which involve many interdependent modules, team members use instant messaging to inform each other about implementation progress. Our findings on this extensive use of chat messaging are similar to those of Dittrich and Giuffrida [14].

Social media are also widely used for knowledge sharing purposes among co-located teams. Our study has shown us that this is particularly true for large organisations where there are many teams in different parts of the same building. Members of the different teams use forums to maintain awareness of the on-going work. Forums are also used to inform other teams about novel solutions to problems or new technology that has proved to be useful in projects. In more urgent situations, stakeholders also use instant messaging or IRC $\mathrm{C}_{13}$ to ask for help or clarifications on possible solutions when they encounter problems. Again our findings support those of Dittrich and Giuffrida [14].

In projects which involve in-house software application development, social media are used to interact with external stakeholders to elicit requirements and for validation and testing purposes. Some of the teams in our survey used Skype ${ }^{\mathrm{TM}}$ to interact with the external stakeholders in these cases. Others used MSN messenger 14 which enables desktop sharing to help end users with live demonstrations.

Our study found that social media are also useful for networking and team building for co-located stakeholders, who tend to use social media to connect to other team members to keep in touch with them. Social media also facilitate the less formal interactions that are essential to team building. For instance, team members use instant messaging and forums to joke and socialise. We found evidence for this in our survey, which revealed that co-located stakeholders interact with each other in two ways. First, they communicate in a formal way with each other about urgent technical issues that require quick responses and secondly they communicate in an informal way to share new ideas, or (in a more social capacity) to team build or network. The importance of both formal and informal reporting are also reported in [15] and in Chapter 12 (Beecham). We found that instant messengers in general and Skype ${ }^{\mathrm{TM}}$ in particular are the preferred social media in the first case as they have high social presence while blogs and forums are widely used in the second case.

\footnotetext{
12 https://github.com/

$13 \mathrm{http}: / /$ www.mirc.com/jarkko.html

$14 \mathrm{http}: / /$ windows.microsoft.com/en-GB/messenger/home
} 


\subsubsection{Results: Benefits and Limitations of Social Media in co-located interactions}

According to our study, social media is very beneficial to software projects. The fact that social media enable stakeholders to reach a very large audience is one of the major benefits. Team members can easily interact through social media with other colleagues who are beyond their own physical "cluster", perhaps being located on a different floor of the building or in a different common area. Thus, social media has broken physical boundaries among stakeholders in the same location facilitating exchange of information among a potentially large number of co-located stakeholders.

Another benefit of social media in this context is that it enables team members who are normally less likely to interact with others to do so. This is particularly true for introverted team members who may find it hard to approach other team members for help or advice.

However, a concern about the use of social media is the ease with which stakeholders can shift from work oriented interactions to more personal ones. One of our survey participants mentioned that social media were not used for co-located interactions because management feared that team members would spend time on unproductive interaction. Social media are not infallible. For example, since there is less regulation on the types of interactions permitted among co-located stakeholders, harmful activities such as trolling (in which team members harass others by publishing inappropriate personal information) can occur resulting in a decrease in performance and a decrease in motivation for the victims [16]. As pointed out in chapter 14 (Beecham) motivation plays an important role in the production of high quality software.

Through the use of social media, team members who are popular may become powerful and hence have greater influence on others. This is especially true in the case of asynchronous social media such as blogs (Twitter, Facebook etc.) and often happens when stakeholders flood these media with an excess of information. For example, if a stakeholder frequently posts irrelevant information on Twitter, other connected stakeholders may become overwhelmed and consequently inadvertently overlook other relevant information from an infrequent poster. The danger is that very active team members can persuade others to accept solutions or technologies that may not necessarily be the best solution for the project. The reverse may also occur: a stakeholder who has very good ideas and suggestions for the project may be completely ignored if their social media activity is overshadowed by very active stakeholders [16].

\subsubsection{Results: Interaction among stakeholders in distributed locations}

Social media are particularly popular when software project teams are globally distributed. All the teams in our study use social media actively to interact with stakeholders who are not in the same location as they are. These can be internal stakeholders such as 
team members who are working in offshore or onshore offices and external stakeholders who are not in the same location as the main software development team. The main software development team is assumed to be located at the premises where the project is being managed. Figure 6 illustrates the outcome of our study concerning how social media are used among distributed stakeholders. This shows that as expected, the main purpose of social media is to facilitate software development activities. The actual activities depend on the role of the stakeholders in the projects.

In the case of internal stakeholders, social media facilitate each phase of the software development process from design through to maintenance. Instant messengers including Skype ${ }^{\mathrm{TM}}$ are used to discuss important issues regarding design and implementation. Before commissioning a release of the software, there is in-house testing. During this exercise, testers record any bugs or feature improvements on forums and blogs and discuss these with other team members using Skype ${ }^{\mathrm{TM}}$ and instant messenger clients. During maintenance, the different teams involved in the software project use social media to decide whether new features or enhancement of existing features are needed. In this phase, we once again found instant messengers (including Skype ${ }^{\mathrm{TM}}$ ) to be the most popular means of communication.

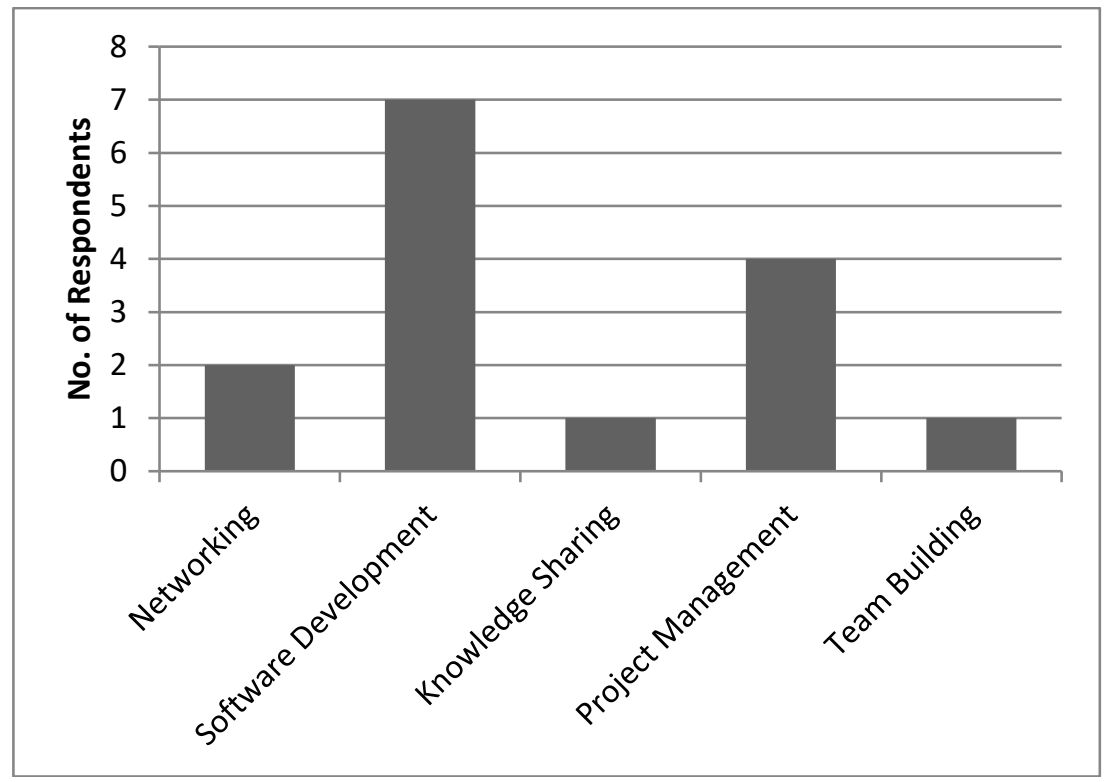

Fig. 6. Types of Interactions among Distributed Stakeholders

Project managers from onshore teams communicate with offshore teams via social media to follow progress and discuss issues during the projects. Our study found that teams which operate with this configuration have regular meetings via instant messengers including Skype ${ }^{\mathrm{TM}}$ to inform teams in other locations about how the project was 
progressing, discuss task allocations and negotiate schedules of delivery. We found that these meetings are held daily or weekly depending on the needs of the projects.

One of the drawbacks of working with offshore teams is the difficulty of interaction which is exacerbated by distance. People in different locations do not have the opportunity to have informal meetings or interactions. This can greatly impact team building and motivation. This can be overcome (at least in part) by using social media. For example, members from some of the teams we surveyed use instant messaging and IRC to have less formal conversations and to get to know other team members better. The ability to initiate conversation among team members who have not been introduced to each other is an important success factor for global projects as it greatly facilitates communication.

Our study has shown that internal stakeholders and external stakeholders who do not share the same physical location frequently interact using social media for software development purposes. Internal stakeholders elicit requirements from external stakeholders using Skype ${ }^{\mathrm{TM}}$, Facebook, forums and blogs. Such social media also support the process of recommending stakeholders that the project team may need. During testing, external stakeholders can use forums and blogs to report bugs and request new features. This also happens during the maintenance phase. According to our study, external stakeholders are often informed about the state of the project via social media. Project managers from the software team tend to use Skype ${ }^{\mathrm{TM}}$ to communicate regularly with external stakeholders about progress, changes to milestones and delivery dates for the project.

Interactions between stakeholders at different locations can require a quick response (typically immediate solutions to design and implementation issues). This is also true when stakeholders are negotiating or providing their requirements for the project. Thus, the use of social media such as instant messengers including Skype ${ }^{\mathrm{TM}}$ ensures that the right social presence and media richness is achieved. The parties involved in these activities need to be able to exchange a large amount of information to ensure that there is no confusion or misunderstanding about what is being discussed. However, there are some interactions which can occur asynchronously without the need for media richness. Examples of such interactions in this context include bug reports and feature requests. In this case, since we require a lower social presence and media richness, social media such as forums and blogs are appropriate.

\subsubsection{Results: Benefits and Limitations of Social Media in distributed interactions}

Social media can be extremely beneficial for interactions among distributed stakeholders. Many of the challenges that are related to distributed software projects can be ameliorated to some extent by social media. Geographical distance whether small or large has a great impact on the ability of teams to collaborate successfully [17].

One of the major issues that social media helps to address is the difficulty of coordination due to independencies and process non-conformities [7, 18]. Our study has 
found that stakeholders in different locations actively use social media to coordinate software implementation. Using social media they are able to communicate any interdependencies that may exist among the components of the software being developed very effectively [19]. The fact that they use social media also helps to align processes in the project. For example, if the stakeholders use specific terminologies in a given process in the development lifecycle, they can discuss this and use agreed terminology that all parties will understand. Slips in schedules $[7,18]$ can also be detected earlier limiting the consequences these may have on other participating teams and stakeholders. Since project managers use social media to do task allocations and follow-ups, any delays can be discovered at an early stage and dealt with as necessary.

Other challenges in distributed software development are mostly associated with offshore software development, mainly because communication difficulties are a major problem. The different cultures and languages of some stakeholders may lead to misunderstandings [7] as also discussed in chapter 12 (Beecham). Furthermore, rapidly changing teams and projects in this context can mean that stakeholders do not have time to form relationships with other stakeholders as also discussed in Chapter 10 (Smite). Our study showed that social media can greatly help in these cases. For example, interacting with a colleague via a video channel can improve understanding through attention to additional subtle cues such as voice tone and facial expressions. Social media also enable stakeholders who have worked together on projects (even for a brief period) to keep in touch via blogs. These stakeholders can eventually become peers who can act as consultants on future projects.

Social media are also useful when managing unstable projects where informal communication is essential [20,21]. For example, in a project where requirements are always changing, social media can support distributed stakeholders by providing them with a rich platform to discuss and interact with each other to resolve issues and project risks. With the help of forums and blogs, stakeholders can also become acquainted with the experts on the various subjects related to the project across the different sites and can thus reach the right expert quickly when necessary [22].

Distributed teams with unstable projects can be managed by using social media in a more formal way in which all interactions are self-moderated by stakeholders. In such a formal setting trolling and flooding are less likely to occur [12]. However, our study found that, as with co-located teams, there is still the risk that using these social media may give rise to less productive work. This can be especially harmful in offshore teams as billing is done by the hour or day spent on the project. Onshore companies or clients will only pay for additional time spent on a given task if this can be justified.

As with all technology, users need to be motivated to use social media. This is the case for the use of social media in offshore teams where some stakeholders cannot see the immediate benefit of adopting social media in their day-to-day tasks and so consider it to be an overhead in their work which is hard to justify in terms of cost. Such nonconformity in the use of social media can mean that social media are regarded as a burden because they may not provide complete information to the stakeholders who are actively trying to use them.

Another issue that can arise specifically from the use of asynchronous social media is the fact that information can become out-of-date before any attention is given to it or 
information may be duplicated. Our study found that for the testing phase (for example) users tend to report the same bug in different ways many times. This adds an overhead to the project as project managers need to analyse each bug report and determine whether the bug has been reported previously.

\subsubsection{Results: Interaction with peers external to the office}

Interactions with peers can have a considerable impact during software projects. The teams in our survey define these peers as being past project team members, colleagues, friends and academics. These peers provide a valuable source of knowledge and information that stakeholders can consult in order to keep in touch with best practice, technologies and trends. Figure 7 illustrates how our participants use social media to interact with their peers.

Networking is the main reason for using social media to interact with peers. In our study we found that internal stakeholders use social media frequently to keep in touch with peers. These relationships have been forged by previous experiences and need to be maintained somehow when the parties are no longer interacting on a day-to-day basis. Social media such as LinkedIn and Facebook enable colleagues to keep in touch by providing updates about events that they post online.

Some of the participants in our survey mentioned using social media to communicate with peers for self-actualisation purposes. Since the field of IT is so vast and new technologies abound, it can be hard to be constantly aware of new tools and techniques. Stakeholders thus use blogs maintained by peers to discuss new technologies. Our study found that stakeholders also use social media to seek technical help from their peers. Often, when they come across an issue in the project they are working on, they either post a question on their own blogs and wait for responses from peers or find forums online where experts can help. Stakeholders also use social media such as blogs and forums to provide reviews on technologies, hardware and methodologies they have used in previous software projects. 


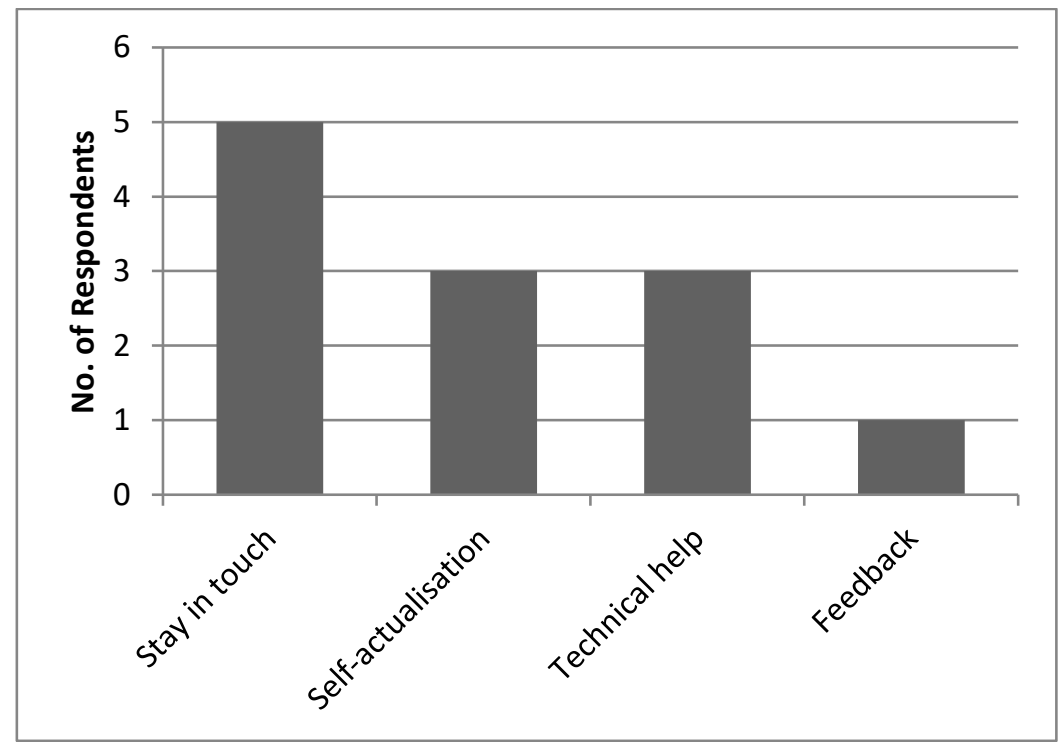

Fig. 7. Types of Interactions among Stakeholders and Peers

These interactions are often done in the stakeholders' spare time and are very informal. Such interactions require low social presence and a reduced level of media richness. Thus blogs and forums are the most common social media used for this. We also found that some teams use instant messaging and Skype ${ }^{\mathrm{TM}}$ at times when they need urgent technical help in projects if relevant peers can be contacted via these media.

\subsubsection{Results: Benefits and Limitations of Social Media in interactions with peers}

The main benefit of interactions between internal stakeholders and their peers is the fact that they can access a wealth of information that can be the key to the success of the software project. By staying in touch with the latest tools and techniques in the field of IT these stakeholders are able to bring innovation into the process of software development. Furthermore, project managers and team members are able to access a larger audience when seeking help. This can lead to numerous solutions for any problems which arise. Managers can then choose the one that best suits their purpose.

However, if stakeholders spend too much time on such informal interactions, this can impact their productivity. The information available from peers may not be entirely appropriate or correct and if stakeholders rely on it and use it unquestioningly in software projects they may introduce more problems than they solve. 


\subsubsection{Results: Impact of Social Media on productivity in Software projects}

Our study found that social media led to an increase in productivity for all our survey participants. The main reason for this is the fact that social media such as Skype ${ }^{\mathrm{TM}}$ enables stakeholders to get responses almost immediately to any queries and on-going discussions. Thus, despite a distributed team, stakeholders can set up meetings almost instantaneously and resolve issues quickly. This means that the teams can make the most of the time allocated to the project and so improve productivity.

In many social media, the basic features such as blogging, instant messaging and video calls are free for use. Furthermore, most social media enable some level of customization. These features are the ones that stakeholders involved in software projects desire most frequently. The low cost and easy customization of social media imply that software engineering companies using them have a set of very powerful tools that are virtually free. Our study confirms that telephone calls and faxes among distributed software teams can be completely eliminated in most cases. This decreases the overhead costs of projects considerably.

\subsubsection{Results: Social Media as a Facilitator in Software Projects}

Half of the participants in our study reported that social media are useful when applying the software methodologies prescribed by their organization or the organization of their customers. The methodologies mentioned included agile methods (See Chapter 13 (Dybå)) and CMMI15. These necessitate good planning, control and monitoring. Such activities can be very tedious to apply across distributed teams as project managers need to get regular updates on the progress of the projects to be able to perform these tasks. Social media provide a convenient way to achieve this. For example, in projects where teams use blogs to provide status updates and make task allocations, project managers can easily be informed about the state of projects by checking status updates and task completion progress.

\subsubsection{Social Media in the Agile Context}

Our study revealed that social media is very popular with all the agile practitioners who responded to our questionnaire. For example, for many of these practitioners, social media are useful for daily stand-up meetings or for demonstrations of prototypes in rapid prototyping.

Agile methods involve working with requirements that are often immature and ambiguous. Short development cycles and efficient communication help to manage such

15 http://cmmiinstitute.com/resources/ 
requirements to reduce the likelihood of defects [23, 24]. Communication and feedback among the stakeholders of an agile project are particularly crucial for its success. For example, the most common agile practice, extreme programming, advocates the practice of real customer involvement where stakeholders take part in weekly or quarterly meetings for exchange of information and planning [25]. Social media are very good tools to promote these kind of activities in the agile context.

Agile methods encourage face-to-face communication to enable the stakeholders to share as much information as possible; this can be helped by facial expression or other cues as well as verbal communication. Thus, in this context, the usefulness of the social media tends to increase with the level of media richness and social presence it provides. For example, according to Wallace et al. [26], videoconferencing is a very good alternative when stakeholders are not available on site for user story explanation. Korkala et al. [27] further elaborates on this to include all instances where effective communication with a remote stakeholder is required.

Social media provide the additional benefit of leaving logs and traces. This can be extremely useful in the rapidly evolving agile context to provide a record of how a project has progressed over time. Such information can be hard to document methodically, especially in large projects with very short development cycles. For example, one of our participants who uses an agile process stated that keeping logs of chats has proved to be very useful when negotiating and justifying billing with customers. Social mining techniques can be used with the resulting logs and traces to get insights into the project dynamics and trends as discussed later in section 16.6.

\subsubsection{Social Media and the Project Manager}

Project complexity and uncertainty are the main challenges that project managers face (Chapter 13 (Dybå), Chapter 8 (Stamelos)). This is especially true in the context of distributed software development. Social media can empower project managers to manage these complexities and uncertainties both proactively and reactively as they arise in a project. However, our understanding of the role of social media for the management of software projects is far from complete [28]

Large distributed software teams involve challenges arising from temporal, geographical and socio-cultural distance [29] that project managers need to overcome. Social media help to coordinate and motivate these teams by promoting communication among the different stakeholders. Project managers can thus have better control of the progress of the individual team members as well as the project as a whole and so can better report to other stakeholders on the status of the project. For example, one of the participants of our study who works with distributed teams emphasized the usefulness of weekly meetings with offshore team members via Skype ${ }^{\mathrm{TM}}$ to plan and assign tasks to the individual team members and set milestones. These weekly meetings also enabled team members to raise any issues that they have encountered or that might arise. Such interactions enable project managers to efficiently tackle these issues to decrease their impact on the project. Our findings are again similar to those of Dittrich and Giuffrida [14]. 


\subsubsection{Threats to Validity}

The validity of this study is limited in a number of ways [30]. The threats to construct, internal and external validity are discussed here.

Construct validity asks whether the variables used in the study accurately measure the concepts they purport to measure. The main threat is posed by participants who may not undertake the survey with sufficient care. For example, participants who are short of time may have simply always chosen to tick the first box presented. However we believe that all participants were well motivated, as they were not under any duress to participate. Consequently it seems likely that the data collected has been submitted accurately and in good faith.

Internal validity is concerned with whether the relationship between the survey outcomes and the use of social media is a causal relationship rather than one which occurred simply by chance. Survey instruments are always subject to a query concerning interpretation: did the participants interpret the questions as they were intended? We had performed trials with the survey within our research group and improved the survey as a result. It is very difficult to determine whether or not every participant interpreted every question in exactly the way that we intended. However we believe that we did constrain possible misinterpretations of the questions as far as possible.

External validity asks whether the results can be generalized. We must acknowledge that the results of our survey are limited due to the small number of companies surveyed. In the fullness of time we hope to repeat this survey with a larger sample. It is also possible that the element of selection (via personal contact) has introduced response bias. However, the personal contacts were often only used in the first instance; the participants who completed the survey were usually unknown to the authors. Our response rate was $39 \%$, which is not unreasonable for such a survey.

\subsection{The Future of Social Media in Software Projects}

Collaborative platforms are increasingly being used by software teams. Collaborative development environments (CDEs) provide teams with the tools to program, debug, refactor and reuse code together with the collaboration tools needed for distributed software development [31]. These collaboration tools usually include social media such as wikis, instant messenger clients and forums. Examples of CDEs include the Jazz $\mathrm{CDE}_{16}$, Redmine 17 and Microsoft Team Foundation Server 18 . Jazz allows developers to perform the usual tasks in software development and adds a web interface to allow developers to interact with other stakeholders via a chat client and access other collaboration information such as bug tracking and project status reports. Microsoft Team

\footnotetext{
$16 \mathrm{http} / / \mathrm{www}-01 . \mathrm{bbm} . \mathrm{com} / \mathrm{software} / \mathrm{rational} / \mathrm{jazz} /$

$17 \mathrm{http}: / /$ www.redmine.org/

$18 \mathrm{http}: / / \mathrm{msdn} . \mathrm{microsoft} . c 0 m / \mathrm{en}-\mathrm{gb} / \mathrm{vstudio} / \mathrm{ff637362.aspx}$
} 
Foundation Server is very similar and enables stakeholders to track a work item or collaborate using a project portal while Redmine provides a web interface where stakeholders can manage projects and collaborate using feeds, wiki and forums. Feeds can provide subscribers with aggregated updates from websites with the latest content. In software projects, feeds are an efficient way to provide awareness about workspaces, developers and processes.

With the advent of disciplines such as social media analytics [32] and social media data mining [33] we expect social media to play a larger and more important role in software projects. Social data mining identifies situations in which groups of people are generating electronic records of documents such as posts, instant message logs and blogs among other documents as part of their normal activity. Potentially useful information implicit in these records can be identified via specific automated computational techniques which harvest and aggregate this information. The results of these operations are then presented for use in a meaningful way [34].

Since social media are widely used in software development projects mining the resulting data can provide valuable insights concerning the most important stakeholders in these projects. This can enhance the ability of social media to help other stakeholders identify experts involved or potential involved in a project. This kind of mining has been successfully applied to code and emails for Postgres [35] where information extracted was used to demonstrate who the more active participants were in the project.

Crowdsourcing is an interesting phenomenon [36] that has great potential as it enables large numbers of stakeholders to provide requirements for new features and to provide feedback on bugs. Crowdsourcing is defined as the act of a company or institution of taking a function once performed by employees and outsourcing it to an undefined and usually large network of people by issuing an open call. The function can be performed by peer-production (in which case the job is performed collaboratively) or by sole individuals. The essential prerequisite is the use of the open call format and the large network of potential workers [37].

Companies are using sites such as Topcoder 19 to outsource coding tasks to the constituency of developers worldwide. Similarly, some requirements elicitation and stakeholder recommendations are outsourced using tools like Stakesource [38] and OneDesk20 respectively. In this new context, social media will become critical for the success of the software projects. Companies who will be crowdsourcing tasks in the software development lifecycle will have a more compelling need to communicate with the successful bidders for the tasks. These bidders can be located in any part of the world. Thus project managers will need to be able to communicate both synchronously (in urgent situations) and asynchronously to make sure that process alignments are performed and the bidders understand what the requirements are. They also need to be able to properly monitor and coordinate projects as such models of operation have many risks associated with them. Social media are excellent as facilitators in this case as they can provide all the required tools for these project management activities.

19 www.topcoder.com/

20 www.onedesk.com/ 


\subsection{Summary and Conclusions}

Social media have already proved their usefulness in software projects in the $21^{\text {st }}$ century. They help project managers to overcome project management difficulties that arise from the relatively new context of distributed software development while providing other project stakeholders with the tools they need to interact successfully with each other. Social media have also brought about a new way of working where knowledge acquired can be shared with other teams or peers or help can be obtained not only from individuals within the organisations involved in the projects but also from external peers who are members of the wider networks of the team members, effectively crowdsourcing new knowledge. New applications for Social media are being discovered on a daily basis. These include social media data mining which can be used to find implicit useful information from the data exchanged using social media. As the very nature of software projects evolves with time, social media will also find new applications in these new project configurations. However, despite their numerous advantages as project management facilitators, social media can still present challenges to software projects if they are not used within a properly regulated framework.

\section{Acknowledgments}

The authors would like to thank all of the participants in the survey reported here. This research is funded by the Faculty of Technology, Design and Environment at Oxford Brookes University. The authors are very grateful for this support.

\section{References}

[1] McNab,Christine, "What social media offers to health professionals and citizens," Bulletin of the World Health Organization, vol. 87, no. 8, p. 566.

[2] C. Hawn, "Take two aspirin and tweet me in the morning: how Twitter, Facebook, and other social media are reshaping health care.," Health affairs (Project Hope), vol. 28, no. 2, pp. 361-8, Jan. 2009.

[3] P. Eckler, G. Worsowicz, and J. W. Rayburn, "Social media and health care: an overview.," PM \& $R$ : the journal of injury, function, and rehabilitation, vol. 2, no. 11, pp. 1046-50, Nov. 2010.

[4] W. G. Mangold and D. J. Faulds, "Social media: The new hybrid element of the promotion mix," Business Horizons, vol. 52, no. 4, pp. 357-365, Jul. 2009.

[5] T. Martín-Blas and A. Serrano-Fernández, "The role of new technologies in the learning process: Moodle as a teaching tool in Physics," Computers \& Education, vol. 52, no. 1, pp. 35-44, Jan. 2009.

[6] A. L. Kavanaugh, E. A. Fox, S. D. Sheetz, S. Yang, L. T. Li, D. J. Shoemaker, A. Natsev, and L. Xie, "Social media use by government: From the routine to the critical," Government Information Quarterly, vol. 29, no. 4, pp. 480-491, Oct. 2012. 
[7] J. D. Herbsleb, "Global Software Engineering: The Future of Socio-technical Coordination," in Future of Software Engineering (FOSE '07), 2007, pp. 188-198.

[8] A. Begel, R. DeLine, and T. Zimmermann, "Social media for software engineering," in Proceedings of the FSE/SDP workshop on Future of software engineering research - FoSER '10, 2010, p. 33.

[9] B. W. Tuckman and M. A. C. Jensen, "Stages of Small-Group Development Revisited," Group Organization Management, vol. 2, no. 4, pp. pp. 419-427, 1977.

[10] I. Oshri and J. Kotlarsky, "Realising the Real Benefits of Outsourcing: Measurement Excellence and Its Importance in Achieving Long Term Value," Global Sourcing of Information Technology and Business Processes, vol. 55, pp. pp. 250-270, 2010.

[11] J. Short, E. Williams, and B. Christie, The Social Psychology of Telecommunications. John Wiley \& Sons Ltd, 1976, p. 206.

[12] R. L. Daft and R. H. Lengel, "Organizational Information Requirements, Media Richness and Structural Design,” Management Science, vol. 32, no. 5, pp. 554-571, May 1986.

[13] A. M. Kaplan and M. Haenlein, "Users of the world, unite! The challenges and opportunities of Social Media," Business Horizons, vol. 53, no. 1, pp. 59-68, Jan. 2010.

[14] Dittrich, Yvonne, and Rosalba Giuffrida. "Exploring the role of instant messaging in a global software development project." Global Software Engineering (ICGSE), 2011 6th IEEE International Conference on. IEEE, 2011.

[15] SE Black, R Harrison, M Baldwin, A Survey of Social Media Use in Global Systems Development, Proceedings of the 1st Workshop on Web 2.0 for Software Engineering, Web2SE, ACM/IEEE ICSE 2010, Pages 1-5, May 2010.

[16] P. Matthews and R. Stephens, "Sociable knowledge sharing online: philosophy, patterns and intervention," Aslib Proceedings. Emerald, 27-Jan-2010.

[17] G. Olson and J. Olson, "Distance Matters," Human-Computer Interaction, vol. 15, no. 2, pp. 139 178, Sep. 2000.

[18] A. Mockus and J. Herbsleb, "Challenges of global software development," in Proceedings Seventh International Software Metrics Symposium, 2001, pp. 182-184.

[19] J. D. Herbsleb and R. E. Grinter, "Splitting the organization and integrating the code," in Proceedings of the 21st international conference on Software engineering - ICSE '99, 1999, pp. 85-95.

[20] J. Galbraith, Organization Design. Addison Wesley Publishing Company, 1977, p. 426.

[21] R. E. Kraut and L. A. Streeter, "Coordination in software development," Communications of the $A C M$, vol. 38, no. 3, pp. 69-81, Mar. 1995.

[22] B. Sengupta, S. Chandra, and V. Sinha, "A research agenda for distributed software development," in Proceeding of the 28th international conference on Software engineering - ICSE '06, 2006, p. 731.

[23] K. Beck and C. Andres, Extreme Programming Explained: Embrace Change. First Edition. Addison Wesley, 1999, p. 224.

[24] P. Abrahamson, O. Salo, J. Ronkainen and J. Warsta, Agile software development methods review and analysis. VTT Publications, 2002, pp 107. http://www.inf.vtt.fi/pdf/publications/2002 /P478.pdf (Last accessed: August 2013)

[25] K. Beck and C. Andres, Extreme Programming Explained: Embrace Change. Second Edition. Addison Wesley, 2004.

[26] N. Wallace, P. Bailey, and N. Ashworth, "Managing xp with multiple or remote customers," in Proceedings of the 3rd International Conference on Extreme Programming and Agile Processes in Software Engineering (XP2002), 2002, pp. 134-137.

[27] M. Korkala, P. Abrahamsson, and P. Kyllonen, "A Case Study on the Impact of Customer Communication on Defects in Agile Software Development," in AGILE 2006 (AGILE'06), pp. 76-88.

[28] Storey, Margaret-Anne, et al. "The impact of social media on software engineering practices and tools." Proceedings of the FSE/SDP workshop on Future of software engineering research. ACM, 2010.

[29] H. Holmstrom, E. Conchuir, P. Agerfalk, and B. Fitzgerald, "Global Software Development Challenges: A Case Study on Temporal, Geographical and Socio-Cultural Distance," in 2006 IEEE International Conference on Global Software Engineering (ICGSE'06), 2006, pp. 3-11. 
[30] C. Wohlin, P. Runeson, M. Höst, M. C. Ohlsson, B. Regnell, and A. Wesslén. Experimentation in Software Engineering: An Introduction. Kluwer Academic Publishers, Norwell, MA, USA. 2000

[31] F. Lanubile, C. Ebert, R. Prikladnicki, and A. Vizcaino, "Collaboration Tools for Global Software Engineering," IEEE Software, vol. 27, no. 2, pp. pp.52-55, Mar. 2010.

[32] Daniel Zeng; Hsinchun Chen; Lusch, R.; Shu-Hsing Li, "Social media analytics and intelligence." Intelligent Systems, IEEE 25.6 (2010): 13-16.

[33] Sufian, Ana, and Ranjith Anantharaman. "Social media data mining and inference system based on sentiment analysis." Master's thesis, Chalmers University of Technology, (2011).

[34] L. Terveen and W. Hill, "Beyond Recommender Systems: Helping People Help Each Other," in HCI in the New Millennium, Addison-Wesley, 2001, pp. 487-509.

[35] C. Bird, A. Gourley, P. Devanbu, M. Gertz, and A. Swaminathan, "Mining email social networks," in Proceedings of the 2006 international workshop on Mining software repositories - MSR '06, 2006, p. 137.

[36] T. O’Reilly, "What is Web 2.0: Design Patterns and Business Models for the Next Generation of Software," Communications \& Strategies, vol. First Quarter, no. 1, p. p. 17, 2007.

[37] J. Howe, 'The rise of crowdsourcing.' Wired magazine 14.6 (2006): 1-4.," Wired magazine, vol. 14 , no. 6 , p. $1-4,2006$

[38] S. L. Lim, D. Damian, and A. Finkelstein, "StakeSource2 .0: Using Social Networks of Stakeholders to Identify and Prioritise Requirements," in Proceeding of the 33rd international conference on Software engineering - ICSE '11, 2011, p. 1022 